\title{
Clinical and neuroimaging profile in postpartum hypernatremia
}

\author{
Sagar Badachi', Raghunandan Nadig,,", G R K Sarma ${ }^{3}$, Akash Awati ${ }^{4}$, Thomas Mathew ${ }^{5}$ \\ ${ }^{1,4}$ Assistant Professor ${ }^{2}$ Associate Professor ${ }^{3,5}$ Professor, Dept. of Neurology, St. John’s Medical College Hospital, Bengaluru, \\ Karnataka, India
}

*Corresponding Author:

Email: docnadig@ rediffmail.com

\begin{abstract}
Introduction: Hypernatremia can cause encephalopathy, rhabdomyolysis, and osmotic demyelination. Postpartum hypernatremia is an uncommon occurrence. The present study attempts to highlight clinical and radiological profile of three patients who had postpartum hypernatremia and to provide differential diagnosis of magnetic resonance imaging findings of postpartum hypernatremia.

Aim: To study clinical and radiological profile of three patients who had postpartum hypernatremia and to provide differential diagnosis of magnetic resonance imaging findings of postpartum hypernatremia.

Material and Methods: The present study was conducted in a tertiary care centre in South India between March 2014 to March 2018. Three patients had hypernatremia presenting in postpartum period. Demographic data, clinical details, results of laboratory examinations and neuroimaging details of these patients were recorded.

Result: All three patients had hypernatremia presenting with encephalopathy. Spasticity was seen in all patients. Quadriparesis was seen in two patients. One patient had generalized tonic clonic seizures. Two patients had hypokalemia. All the three patients had Hyperchloremia, Azotemia and raised serum osmolality. Phosphorous levels was high in one patient. Creatine kinase levels was high in one patient. All patients had hypermagnesemia and normal calcium levels. Thrombocytopenia was seen in one patient. On neuroimaging, splenium of corpus callosum, hippocampus and posterior limb of internal capsule were involved in all patients. Cerebral peduncles in two patients. Corona Radiata and cerebellar white matter were involved in one patient.

Conclusion: Early identification and meticulous management of hypernatremia is of paramount importance. Neuroimaging Findings in postpartum hypernatremia is distinct.
\end{abstract}

Key words: Postpartum, Hypernatremia, Encephalopathy, Osmotic demyelination, rhabdomyolysis.

\section{Introduction}

Hypernatremia is a state wherein serum sodium exceeds $146 \mathrm{mEq} / \mathrm{l}^{1}$ It can cause encephalopathy, rhabdomyolysis, and osmotic demyelination. ${ }^{2}$ If not treated meticulously it can have disastrous consequences. Postpartum hypernatremia is an uncommon occurrence. There is paucity of data in literature on its clinical and neuroimaging profile. The present study attempts to highlight clinical and radiological profile of three patients who had postpartum hypernatremia and to provide differential diagnosis of Magnetic resonance imaging findings of postpartum hypernatremia.

\section{Aim}

To study clinical and radiological profile of three patients who had postpartum hypernatremia and to provide differential diagnosis of Magnetic resonance imaging findings of postpartum hypernatremia.

\section{Material and Methods}

The present study was conducted in a tertiary care centre in South India between March 2014 to March 2018. Three patients had hypernatremia presenting in postpartum period. Demographic data, clinical details, results of laboratory examinations and neuroimaging details of these patients were recorded.

\section{Results}

Case 1: A 33 year old lady 20th day of postpartum period presented with altered sensorium, inability to move upper and lower limbs. On examination she had moderate dehydration. She was drowsy, not oriented to time, place and person. Oculocephalic reflex was present. Pupils were $5 \mathrm{~mm}$ briskly reacting to light. She had spastic quadriparesis. Deep tendon reflexes were pathologically brisk. Plantar response was bilaterally extensor. Complete hemogram was normal. Serum sodium levels of $163 \mathrm{mEq} / \mathrm{L}$, potassium of $3.6 \mathrm{mEq} / \mathrm{L}$, Chloride of $136 \mathrm{mEq} / \mathrm{L}$ was noted. Urea was $97 \mathrm{mg} / \mathrm{dL}$, creatinine was $1.13 \mathrm{mg} / \mathrm{dL}$, calcium was $8.2 \mathrm{mg} / \mathrm{dL}$, phosphorous was $4.46 \mathrm{mg} / \mathrm{dL}$ and magnesium was 3.18 $\mathrm{mg} / \mathrm{dL}$. Serum osmolality was $361 \mathrm{mosmol} / \mathrm{kg}$. Neuroimaging showed diffusion restriction in splenium of corpus callosum, hippocampus and posterior limb of internal capsule. T2 Flair showed hyperintensity in the same regions. (Fig. 1). 


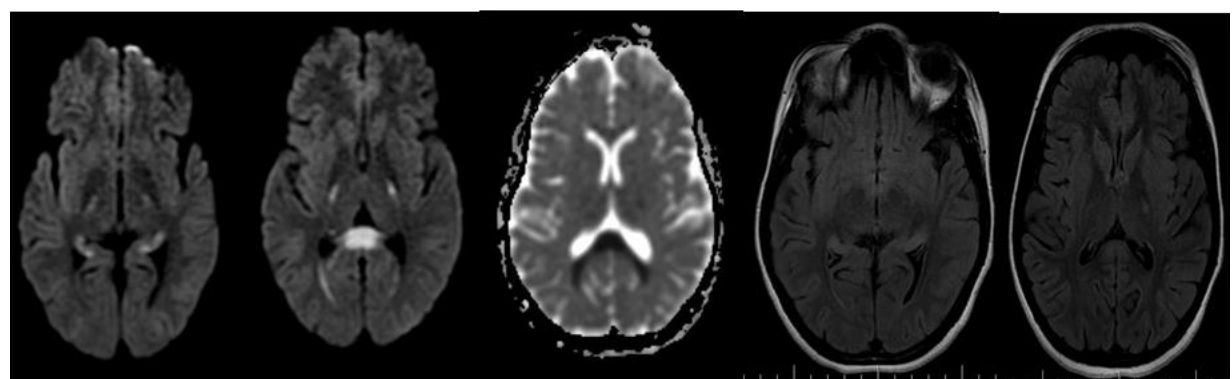

Fig. 1: MRI Brain Showing Diffusion Restriction in Splenium of corpus callosum. Hippocampus and posterior limb of internal capsule. ADC reversal and T2 flair hyperintensity is seen in the same regions

Case 2: A 22 year female presented with generalized tonic clonic seizures on day 8 of postpartum. On examination she was in altered sensorium. She was stuporous. Pupils were $4 \mathrm{~mm}$ and briskly reacting to light. Oculocephalic reflex was present. Spasticity was noted in all limbs, however there was no paucity of movements of limbs. Deep tendon reflexes were pathologically brisk. Bilateral plantar responses were extensor. She had moderate dehydration. Complete hemogram was normal. Serum sodium levels of 176 $\mathrm{mEq} / \mathrm{L}$, potassium of $2.6 \mathrm{mEq} / \mathrm{L}$, Chloride of 143 $\mathrm{mEq} / \mathrm{L}$ was noted. Urea was $89 \mathrm{mg} / \mathrm{dL}$, creatinine was $2.6 \mathrm{mg} / \mathrm{dL}$, calcium was $8.6 \mathrm{mg} / \mathrm{dL}$, phosphorous was $4.26 \mathrm{mg} / \mathrm{dL}$ and magnesium was $4.26 \mathrm{mg} / \mathrm{dL}$. Serum osmolality was $391 \mathrm{mosmol} / \mathrm{kg}$. Neuroimaging showed diffusion restriction in splenium of corpus callosum, hippocampus, posterior limb of internal capsule and cerebral peduncles. T2 Flair showed hyperintensity in the same regions. (Fig. 2)

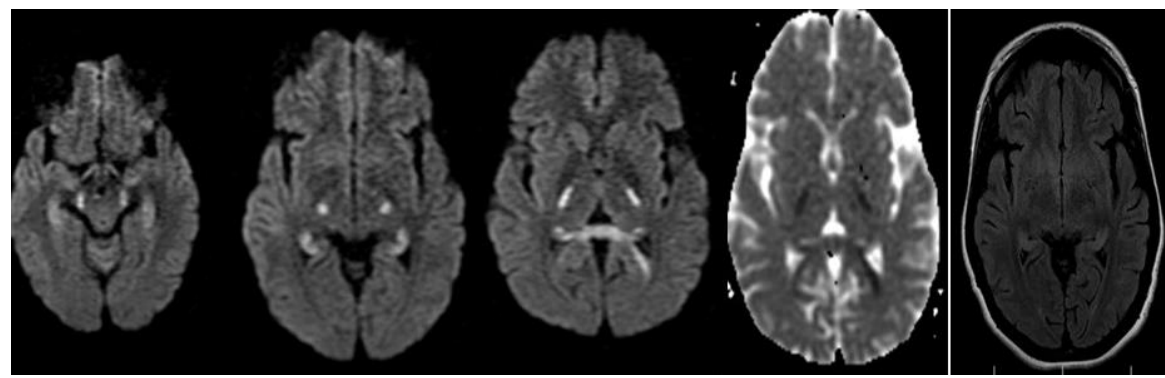

Fig. 2: MRI brain shows diffusion restriction in splenium of corpus callosum, hippocampus, posterior limb of internal capsule and cerebral peduncles. ADC reversal and T2 flair hyperintensity in the same region

Case 3: A 23 year old female presented with altered sensorium, inability to move limbs and two episodes of generalized tonic clonic seizures on day 12 of postpartum. She was stuporous. Pupils were $3 \mathrm{~mm}$ and briskly reacting to light. Oculocephalic reflex was present. Spasticity was noted in all limbs. There was paucity of movements of all limbs. Deep tendon reflexes were pathologically brisk. Bilateral plantar responses were extensor. She had moderate dehydration. Complete hemogram revealed thrombocytopenia (platelet count of 1.2 lakhs). Serum sodium levels of $182 \mathrm{mEq} / \mathrm{L}$, potassium of $2.7 \mathrm{mEq} / \mathrm{L}$, Chloride of $149 \mathrm{mEq} / \mathrm{L}$ was noted. Urea was $84 \mathrm{mg} / \mathrm{dL}$, creatinine was $1.6 \mathrm{mg} / \mathrm{dL}$, calcium was $8.8 \mathrm{mg} / \mathrm{dL}$, phosphorous was $6 \mathrm{mg} / \mathrm{dL}$ and magnesium was 4.06 $\mathrm{mg} / \mathrm{dL}$. Serum osmolality was $401 \mathrm{mosmol} / \mathrm{kg}$. Neuroimaging showed diffusion restriction in splenium of corpus callosum, hippocampus, posterior limb of internal capsule, cerebral peduncles, cerebellar white matter and corona Radiata. T2 Flair showed hyperintensity in the same regions. (Fig. 3) 


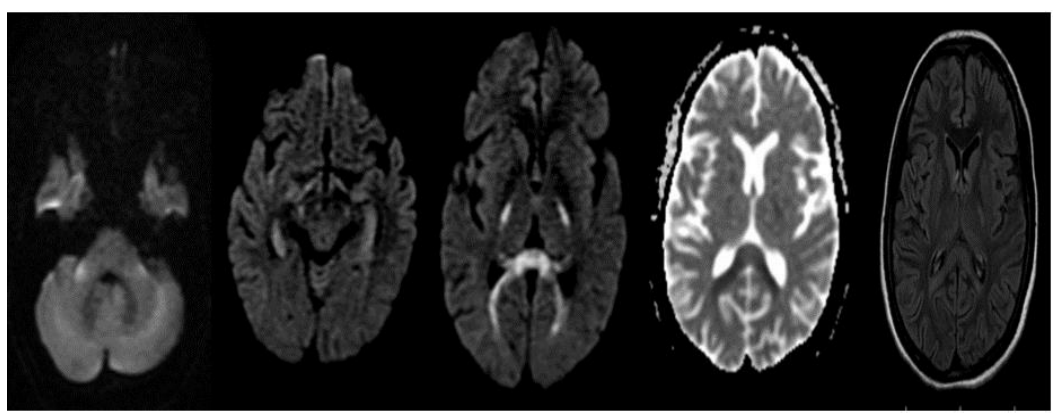

Fig. 3: MRI brain shows diffusion restriction in splenium of corpus callosum. Hippocampus, posterior limb of internal capsule, cerebral peduncles, cerebellar white matter and corona radiata. ADC reversal and T2 flair hyperintensity is seen in the same region

All patients were treated with infusion of hypotonic intravenous fluids using $0.45 \%$ saline and $5 \%$ dextrose with potassium supplements as required. Plain water was given through nasogastric tube. Once the sensorium improved, the patients were given salt restricted feeds with continuation of intravenous fluids as dictated by the serum sodium levels. There was significant improvement after symptomatic and supportive care.

\section{Discussion}

Hypernatremia is a state of electrolyte imbalance in which the serum level of sodium exceeds $146 \mathrm{mEq} / \mathrm{l}$. Most common cause of hypernatremia is due to volume loss secondary to vomiting, diarrhea or water restriction. The incidence of spontaneous hypernatremia is less common. Hypernatremia can present with encephalopathy, seizures and focal neurological deficits caused due to osmotic demyelination. The recommended rate of reduction of serum sodium should not exceed $2 \mathrm{mEq} / \mathrm{l} / \mathrm{h}$ with appropriate selection of fluids .The mortality rate can be as high as $75 \%$ in acute hypernatremia and with chronic hypernatremia mortality rate approximates $10 \%$ when the serum sodium exceeded $160 \mathrm{mEq} / 1 .^{3}$

All 3 patients presented with encephalopathy in the summer season. Fluid intake was restricted and warm clothing was provided in all our patients as a part of traditional postpartum care. Hypernatremia in our patients could be due dehydration resulting from combined effects of increased perspiration owing to rise in ambient temperature in the summer season, warm clothing and decreased fluid intake.

Two patients out of three had hypokalemia. Hypernatremia with hypokalemia has been reported in patients with adipsic hypernatremia. ${ }^{4}$ It is also reported in a patient resulting from hunger strike. ${ }^{5}$ In a case series of 11 patients, the serum potassium levels were low in seven and normal in four patients in a study conducted by Naik et al. ${ }^{2}$

The cause of hypokalemia in our patients is not clear. Neuroimaging did not reveal abnormalities in the region of hypothalamus, pituitary and infundibulum.
All the 3 patients had hyperchloremia, azotemia and raised serum osmolality. Naik et al noted hyperchloremia, azotemia and raised serum osmolality in all patients. The cause of the kidney dysfunction in these patients can be multifactorial secondary to poor water intake and fever complicated by severe rhabdomyolysis.

Phosphorous levels was high in one patient. Creatine kinase levels was high in the same patient. Hypernatremia has been associated with rhabdomyolysis and profound elevation of the serum CK levels. ${ }^{6}$ Naik et al reported elevated serum CK levels in nine out of eleven patients. ${ }^{2}$ Elevated cretine kinase level in postpartum hypernatremia is also reported by Bhatia et $\mathrm{al}^{7}$ and Suri et al. ${ }^{8}$

All our patients had hypermagnesemia which can be explained by acute kidney injury. All patients had normal calcium levels.

Thrombocytopenia was seen in one patient. The cause of thrombocytopenia is not clear. Naik et al reported thrombocytopenia in 9 out of 11 patients etiology of which was not clear. ${ }^{2}$ Thrombotic thrombocytopenic purpura (TTP) consists of pentad of microangiopathic hemolytic anemia, thrombocytopenic purpura, renal dysfunction, neurological manifestations and fever. It can be seen during pregnancy as well as in puerperium. ${ }^{9}$ Our patient did not fulfil the pentad as she did not have fever and her indirect bilirubin levels were normal.

Neuroimaging findings in our patient are distinct. Such findings are not reported in TTP patients. Cerebral imaging in 12 patients of TTP revealed features of posterior reversible encephalopathy syndrome (PRES) in seven, ischemic strokes in three and hematoma in one patient. ${ }^{10}$ MRI findings in 33 cases of TTP showed $82 \%$ abnormality with nearly half of them demonstrating PRES and remaining patients having infarcts. ${ }^{11}$

Neuroimaging in the present study revealed involvement of splenium of corpus callosum, hippocampus and posterior limb of internal capsule in all patients. Cerebral peduncles involvement in two patients. Corona radiata and cerebellar white matter were involved in one patient. 
Cerebral shrinkage is one of the features in neuroimaging studies and is shown to be reversible with adequate correction of hypernatremia and hydration. ${ }^{12}$

Hyperintensities were reported in externalcapsule, cerebellum, hippocampus, lateral thalamic nuclei and subcortical white matter in T2 weighted imaging. In three children with hypernatremia. ${ }^{13}$

Hyperintensities in corpus callosum, external capsules and thalamus was seen in a child with hypernatremia. ${ }^{14}$

In a case series of 11 patients Naik et al reported involvement of splenium in all patients. 9 patients had internal capsule involvement. Hippocampus, lateral thalami and corona radiata involvement was seen in 7 patients. Cerebellar peduncle involvement was seen in 6 patients. Crus cerebri was involved in 5 patients. Pons and cerebellum involvement was seen in 4 patients. External capsule was involved in 1 patient. $^{2}$

Wine-glass"-appearance is described in patients with hypernatremic myelinolysis. ${ }^{15}$ It consists of extensive symmetrical T2, FLAIR, and DWI hyperintensities of white matter, internal capsule through midbrain and pons to middle cerebellar peduncle. It depicts the involvement of corticospinal tract. Symmetrical hyperintensities of internal capsule, crus cerebri, and pons on coronal T2W images are seen. Similar appearance is described in entities like primary lateral sclerosis, amyotrophic lateral sclerosis (ALS), and leukodystrophies.

Osmotic demyelination can be seen in rapid correction of hyponatremia.MR imaging findings of osmotic demyelination syndrome consist of abnormal hyperintensity involving pons and extrapontine sites including basal ganglia, thalami, and cerebral white matter. Symmetric trident-shaped hyperintensity in the central pons is a hallmark finding with sparing of ventrolateral pons and the pontine portion of corticospinal tracts. Typical sites of involvement of extrapontine myelinolysis associated with rapid correction of hyponatremia include caudate nucleus, lentiform nucleus, and thalami. The lesions exhibit minimal to no mass effect and show no enhancement in most of the cases. Restricted diffusion is seen in most of the cases. Atypical sites of involvement include grey matter, white matter, corpus callosum, splenium, cerebellum, hippocampus, and external capsule. Other rare sites include midbrain, subthalamic nuclei, claustrum, hypothalamus, medulla, and amygdala. ${ }^{16}$

Metronidazole poisoning manifests after metronidazole intake for more than 2 weeks. MR imaging of brain demonstrates abnormal symmetrical hyperintensity within cerebral white matter, corpus callosum, and cerebellum. Near total resolution of findings is seen on discontinuation of medication. ${ }^{17}$

Acute disseminated encephalomyelitis [ADEM] presents with brain lesions predominantly in cerebral or cerebellar cortices, subcortical and parieto-occipital white matter, centrum semiovale, cerebellar peduncles, and the brainstem. The lesions are large, asymmetric, irregular in morphology, and decrease dramatically with short course of steroids, immunoglobulins, or plasmapheresis. Associated involvement of spinal cord can also be seen. ${ }^{18}$

Posterior reversible encephalopathy syndrome (PRES) is seen in patients with hypertensive encephalopathy, pregnant patients with eclampsia, and post-transplant population on cyclosporin A and tacrolimus, uremia, thrombotic thrombocytopenia purpura, and hemolytic-uremic syndrome. Neuroimaging typically show symmetrically distributed areas of vasogenic edema predominantly within posterior circulation territories involving occipital and parietal lobes.Involvement of anterior circulation structures can also be seen in variant forms. The abnormalities primarily affect white matter, although cortex may also get involved. Localized mass effect and mild enhancement may be seen. ${ }^{19}$

Cerebral venous thrombosis is another differential diagnosis associated with peripartum state. The absence of a flow void and the presence of altered signal intensity in the sinus is a hallmark finding of sinus thrombosis on MR images. Parenchymal lesions, focal edema, venous infarcts, and hemorrhages can be demonstrated on neuroimages. ${ }^{20}$

\section{Conclusion}

Postpartum hypernatremia is an uncommon condition. It is potentially lethal metabolic dysfunction which can produce encephalopathy, osmotic demyelination and rhabdomyolysis. Early identification and meticulous management of hypernatremia is of paramount importance to avert disastrous consequences. Neuroimaging findings in postpartum hypernatremia is distinct. Conditions like cerebral venous thrombosis, PRES, ADEM are to be considered in differential diagnosis. Further studies are needed to elucidate the pathogenesis of hypernatremia in postpartum period. Predilection to affect specific areas of brain needs further research.

\section{References}

1. Pevelsky PM, Bagrath R, Greenberg A. Hypernatremia in hospitalized patients. Ann Intern Med 1996;124:197-203

2. Naik KR, Saroja AO. Seasonal postpartum hypernatremic encephalopathy with osmotic extrapontine myelinolysis and rhabdomyolysis. J Neurol Sci 2010;291:5-11

3. Berl T, Taylor J. Disorders of water balance. In: Fink MP, Abraham E, Vincent JL, Kochanek DM, editors. Textbook of Critical Care Medicine. 5th ed. Philadelphia: Saunders; 2005:1085-96.

4. Blank MS, Farnsworth PB. Idiopathic symptomatic hypernatremia in a 9-year old boy: a clinical and physiologic evaluation. J Pediatr 1974;85:215-9.

5. Van der Helm-van Mil AH, van Vugt JP, Lammers GJ, Harinck HI. Hypernatremia from a hunger strike as a cause of osmotic myelinolysis. Neurol 2005;64:574-5.

6. Lima EQ, Aguiar FC, Barbosa DM, Burdmann FA. Severe hypernatremia $(221 \mathrm{mEq} / \mathrm{l})$, rhabdomyolysis and 
acute renal failure after cerebral aneurysm surgery. Nephrol Dial Transplant 2004;19:2126-9.

7. Bhatia S, Kapoor AK, Sharma A, et al. Cerebral encephalopathy with extrapontine myelinolysis in a case of postpartum hypernatremia. Indian J Radiol Imaging 2014;24:57-60

8. Suri V, Jasuja S, Suri N, Saini R, Naga MK, Suri K. Postpartum hypernatremic encephalopathy with "Wine Glass Sign" on magnetic resonance imaging. Apollo Med 2017;14:188-9

9. Levine SP. Thrombotic thrombocytopenic purpura and other forms of nonimmunological platelet distruction. In: Greer JP, Rodgers GM, Forester J, Paraskevas F, Lukens JN, Glader B, editors. Wintrobe's Clinical Hematology. 11th ed. Philadelphia: Lippincot Williams Wilkins 2005:1555-65.

10. Bakshi R, Shaikh ZA, Bates VE, Kinkel PR. Thrombotic thrombocytopenic purpura: brain CT and MRI findings in 12 patients. Neurol 1999;52:1285-8.

11. Burus TM, Wijdicks EFM, Rabinstein AA. Brain lesions are most often reversible in acute thrombotic thrombocytopenic purpura. Neurol 2009;73:66-70.

12. Machino T, Yoshizawa T. Brain shrinkage due to acute hypernatremia. Neurol 2006;67:112-3.

13. BrownWD, Caruso JM. Extrapontinemyelinolysiswith involvement of hippocampus in three children with severe hypernatremia. J Child Neurol 1999;14:428-33.
14. Achiva S, Ando K, Ishikura R, Takada Y, Takahashi Y. A case of extrapontine myelinolysis precipitated by correction of a hyper-osmolar state. Nippon Igaku Hoshasen Gakkai Zasshi 2004;64:310-2.

15. Saroja AO, Naik KR, Mali RV, Kunam SR. Wine Glass' sign in recurrent postpartum hypernatremic osmotic cerebral demyelination. Ann Indian Acad Neurol 2013;16:106-10.

16. Sajith J, Ditchfield A, Katifi HA. Extrapontine myelinolysis presenting as acute parkinsonism. $B M C$ Neurol 2006;6:33.

17. Ahmed A, Loes DJ, Bressler EL. Reversible magnetic resonance imaging findings in metronidazole-induced encephalopathy. Neurol 1995;45:588-9.

18. Singh S, Alexander M, Korah IP. Acute disseminated encephalomyelitis: MR imaging features. AJR Am J Roentgenol 1999;173:1101-7

19. Diego J. Covarrubias, Patrick H. Luetmer, Norbert G. Campeau. Posterior Reversible Encephalopathy Syndrome: Prognostic Utility of Quantitative DiffusionWeighted MR Images. AJNR Am J Neuroradiol 2002;23:1038-48.

20. Leach JL, Fortuna RB, Jones BV, Gaskill-Shipley MF. Imaging of cerebral venous thrombosis: Current techniques, spectrum of findings, and diagnostic pitfalls. Radiographics 2006;26:S19-41. 